\title{
BestMasters
}


Mit „BestMasters“ zeichnet Springer die besten, anwendungsorientierten Masterarbeiten aus, die im Jahr 2013 an renommierten Wirtschaftslehrstühlen Deutschlands, Österreichs und der Schweiz entstanden sind.

Die mit Bestnote ausgezeichneten und durch Gutachter zur Veröffentlichung empfohlenen Arbeiten weisen i.d.R. einen deutlichen Anwendungsbezug auf und behandeln aktuelle Themen aus unterschiedlichen Teilgebieten der Wirtschaftswissenschaften.

Die Reihe wendet sich an Praktiker und Wissenschaftler gleichermaßen und soll insbesondere auch Nachwuchs-Wissenschaftlern Orientierung geben. 


\section{Dagmar Rankl}

\section{Ressorts im Zeitalter \\ von Medienwandel und \\ Boulevardjournalismus}

Themenwahl als Qualitätskriterium

Mit einem Geleitwort von

Prof. (FH) Dr. Martina Lettner

Springer Gabler 
Dagmar Rankl

Silz, Österreich

ISBN 978-3-658-04514-2

ISBN 978-3-658-04515-9 (eBook)

DOI $10.1007 / 978-3-658-04515-9$

Die Deutsche Nationalbibliothek verzeichnet diese Publikation in der Deutschen Nationalbibliografie; detaillierte bibliografische Daten sind im Internet über http://dnb.d-nb.de abrufbar.

Springer Gabler

(C) Springer Fachmedien Wiesbaden 2014

Das Werk einschließlich aller seiner Teile ist urheberrechtlich geschützt. Jede Verwertung, die nicht ausdrücklich vom Urheberrechtsgesetz zugelassen ist, bedarf der vorherigen $\mathrm{Zu}$ stimmung des Verlags. Das gilt insbesondere für Vervielfältigungen, Bearbeitungen, Übersetzungen, Mikroverfilmungen und die Einspeicherung und Verarbeitung in elektronischen Systemen.

Die Wiedergabe von Gebrauchsnamen, Handelsnamen, Warenbezeichnungen usw. in diesem Werk berechtigt auch ohne besondere Kennzeichnung nicht zu der Annahme, dass solche Namen im Sinne der Warenzeichen- und Markenschutz-Gesetzgebung als frei zu betrachten wären und daher von jedermann benutzt werden dürften.

Gedruckt auf säurefreiem und chlorfrei gebleichtem Papier

Springer Gabler ist eine Marke von Springer DE. Springer DE ist Teil der Fachverlagsgruppe Springer Science+Business Media.

www.springer-gabler.de 


\section{Geleitwort}

Die massenmediale Kommunikation entwickelte sich in den vergangenen Jahrzehnten zum sozialen Totalphänomen. Entsprechend stark wuchs seither das Interesse der Sozial- und Wirtschaftswissenschaften an den Massenmedien, etwa an ihren Inhalten, ihren Zielgruppen, ihren Eigentümerstrukturen sowie ihren personellen Ressourcen. Die gleichzeitige technologische Weiterentwicklung hin zu den so genannten neuen Medien wandelte - in wechselseitiger Abhängigkeit - sowohl die Medieninhalte, die Mediennutzung als auch die Medienlandschaft. Etablierte Printmedien sehen sich durch Online-Medien neuen personellen, strukturellen und ökonomischen Herausforderungen ausgesetzt. Zwar gibt es zahlreiche Untersuchungen zu Printmedien, von Bloggern bis Citizen Journalism, und mittlerweile auch fundierte Analysen des (vorgeblich) journalistischen Online-Angebotes. Wie aber etablierte Printmedien inhaltlich auf die neuen Kanäle reagieren und in ihnen reüssieren, wurde bisher nicht eingehend betrachtet.

Linguistin Dagmar Rankl nahm sich im Zuge ihres „Marketing \& Kommunikationsmanagement“- Studiums an der FH Kufstein Tirol dieser Forschungslücke engagiert an. Ausgehend von einer soliden theoretischen Fundierung verglich sie erst die Startseiten der Onlinepräsenzen zweier österreichischer Tageszeitungen, um so mögliche Unterschiede in der inhaltlichen Gestaltung zwischen Boulevard- und Qualitätspresse im Internet herauszuarbeiten. Die erstaunlichen Ergebnisse bewogen sie zu einer weitergehenden komparatistischen Arbeit, in der sie zusätzlich die jeweiligen gedruckten Titelblätter mit den Startseiten verglich. Die gelungene empirische Herangehensweise sowie die Kontextualisierung der Ergebnisse zeichnen ihre Analyse in besonderem Maße aus. 
Zentraler Befund der ersten Erhebung war, dass die inhaltliche Gestaltung der Startseiten des Boulevard- und des Qualitätsmediums nicht so stark voneinander abweichen, wie dies die Beschreibungen der qualitativen Standards der Printmedien in wissenschaftlichen Texten (diese befassten sich jedoch nur mit der Print-Variante) nahelegen würden.

Hatten sich, abseits bereits publizierter wissenschaftlicher Betrachtung, die Printprodukte jüngst inhaltlich angeglichen? Mögliche Gründe dafür wären mannigfaltig. Dagmar Rankls anschließender Vergleich der Startseiten und Titelblätter der jeweiligen Massenmedien zeigte, dass auch innerhalb eines Mediums die inhaltliche Gestaltung der ersten Seite der Printversion und der Online-Variante nicht deckungsgleich ist. Es näherten sich also nicht die beiden Medien als solche an, vielmehr unterscheidet sich die OnlineVariante von der Printausgabe.

Dagmar Rankl beschreibt in ihrer Arbeit nicht nur aktuelle Entwicklungen, sondern bettet diese zukunftsweisend in den theoretischen Rahmen ein. So wird es möglich, auch Rückschlüsse auf die LeserInnen und damit auf die Zielgruppen und deren Mediennutzungsgewohnheiten zu ziehen. Rankls Arbeit wirft andererseits bewusst weitergehende Fragen auf - die hoffentlich zu ergebnisreicher Anschlussforschung in einem spannenden das Alltagsals auch das Wirtschaftsleben durchdringenden Bereich führen.

Ich wünsche Dagmar Rankl alles Gute für ihre wissenschaftliche Karriere und den LeserInnen ihrer Arbeit eine interessante Lektüre.

Prof. (FH) Dr. Martina Lettner 


\section{Vorwort}

„,Zeitung” ist nicht an Papier gebunden“ - mit diesen Worten greifen Schulz-Bruhdoel und Bechtel in ihrem Werk „Medienarbeit 2.0 - Das Praxisbuch für PR und Journalismus von morgen“ 2009 den Zeitungswandel auf. Bis heute ist diese Thematik ein zentraler Punkt der medienwissenschaftlichen Forschung und gewinnt zunehmend an Bedeutung in verschiedenen Disziplinen - die wachsende Auseinandersetzung mit dem Medienwandel und die Wechselwirkung mit der Gesellschaft und deren Wandelerscheinungen ist viel weitreichender als nur im auf den ersten Blick ersichtlichen Forschungsgebiet.

Diese vorliegende Untersuchung soll dazu beitragen, die Verantwortung des Journalismus als Meinungsbildner für jegliche soziale Schicht zu thematisieren und erneut in Diskussion zu bringen, um Chancen nutzen und Risiken minimieren zu können.

In diesem Zusammenhang soll diese Arbeit einen interdisziplinären Beitrag zum oben genannten Forschungsgebiet und auch Ideen für aufbauende und spezifischere Forschungen liefern.

Anhand einer Inhaltsanalyse der Themen-/Ressortwahl auf den Titelseiten zweier österreichischer Tageszeitungen sollen drei zentrale Phänomene des österreichischen (Print-) Medienmarktes tangiert werden: Durch den Vergleich der Online- und Printversion der Titelseiten beider Zeitungen wird der Online-Journalismus als Chance und zugleich Herausforderung für den traditionellen Journalismus thematisiert. Aufbauend auf diese Ergebnisse greift die Wahl der Analysegegenstände (die beiden Zeitungen Standard und Krone) die Qualitätsdebatte des österreichischen Zeitungsmarktes auf. Die Aufarbeitung dieser Themen soll mit Hilfe der Auswertung der erhobenen 
Daten Grundlage für die Bearbeitung der Thematik der demokratiepolitischen Funktion des Mediums Zeitung liefern.

Die nachfolgenden Untersuchungen stellen zwei getrennt verfasste wissenschaftliche Abschlussarbeiten aus den Jahren 2010 und 2013 dar, die zum besseren Verständnis und für eine erhöhte Leserfreundlichkeit zu einem Text zusammengefasst wurden, um Wiederholungen und Überschneidungen zu verhindern. An dieser Stelle gilt auch ein besonderer Dank meiner Betreuerin Prof. (FH) Dr. Martina Lettner für die beispielhafte Unterstützung und die wertvollen Inputs.

Oktober 2013

Dagmar Rankl 


\section{Inhaltsverzeichnis}

Geleitwort $\quad$ V

Vorwort VII

Inhaltsverzeichnis IX

Abbildungsverzeichnis $\quad \mathrm{XI}$

1. Einleitung 1

1.1. Erkenntnisgegenstand, Forschungsfragen und Ziele 1

1.2. Begründung der Relevanz 5

1.3. Forschungsstand 10

1.4. Aufbau der Arbeit 13

2. Theoretische Grundlagen $\quad 15$

2.1. Journalismus $\quad 15$

2.1.1. „Journalismus“ - ein Definitionsversuch 15

2.1.2. Funktionen und Aufgaben des Journalismus 17

2.2. Zeitung im Medienwandel - Entwicklung der Presse 21

2.3. Online-Journalismus 25

2.3.1. „Online“-Journalismus - ein Definitionsversuch 25

2.3.2. Chancen und Risiken des Online-Journalismus 27

2.4. Qualitätsjournalismus - Boulevardjournalismus 31

2.5. Die Titelseite $\quad 35$

2.6. Die RezipientInnen 36

3. Methodik 39

3.1. Methodisches Vorgehen 40

3.2. Codebuch 41

3.3. Untersuchungsgegenstand $\quad 46$

3.3.1. Die Zeitungen 46

3.3.2. Die Titelseiten 49 
4. Komparatistische Analyse $\quad 55$

4.1. Anzahl der Artikelteaser $\quad 55$

4.2. Ressortaufteilung $\quad 57$

4.3. hard news / soft news $\quad 59$

4.4. Die Leitartikel 60

5. Schlusswort $\quad 61$

Literaturverzeichnis $\quad 65$ 


\section{Abbildungsverzeichnis}

Abb. 1: Benutzerzahlen ausgew. Online-Zeitungen von 1998-2008 30

Abb. 2: Ressortaufteilung der beiden Zeitungen 43

Abb. 3: Variablen des Codebogens $\quad 44$

Abb. 4: Die Homepage-Startseite von www.derstandard.at 49

Abb. 5: Titelseite Standard, 01.Dezember 2011

Abb. 6: Die Homepage-Startseite von www.krone.at 52

Abb. 7: Titelseite Krone, 16.Dezember 2011

Abb. 8: Gesamtanzahl der Artikelteaser - Vergleich 55

Abb. 9: Ressortverteilung Krone $\quad 58$

Abb. 10: Ressortverteilung Standard 58

Abb. 11: hard news \& soft news - Gesamtvergleich $\quad 59$

Abb. 12: Leitartikel Gesamtvergleich $\quad 60$ 